\title{
ANALYSIS OF CMOS AND MTCMOS CIRCUITS USING 250 NANO METER TECHNOLOGY
}

\author{
M Suresh ${ }^{1}$, A K Panda ${ }^{1}$, Mukesh Sukla ${ }^{1}$, Marakonda Patnaikuni Vasanthi ${ }^{2}$ \\ and Sowpati Santhi ${ }^{3}$ \\ ${ }^{1}$ Department of ECE, NIST, Berhampur, Odisha \\ msuresh73@hotmail.com \\ akpanda62@hotmail.com \\ Mukesh_ele02@yahoo.co.in \\ ${ }^{2}$ Department of ECE, RGUKT, Basar, Telangana \\ vasanthimpiitegmail.com \\ ${ }^{3}$ Department of ECE, RGUKT, Nuzvid, Andhra Pradesh \\ santhisowpati555@gmail.com
}

\begin{abstract}
The low-power consumption with less delay time has become an important issue in the recent trends of VLSI. In these days, the low power systems with high speed are highly preferable everywhere. Designers need to understand how low-power techniques affect performance attributes, and have to choose a set of techniques that are consistent with these attributes. The main objective of this paper is to describe, how to achieve low power consumption with approximately same delay time in a single circuit. In this paper, we make circuits with CMOS and MTCMOS techniques and check out its power and delay characteristics. The circuits designed using MTCMOS technique gives least power consumption.
\end{abstract}

All the pre-layout simulations have been performed at 250nm technology on tanner EDA tool.

\section{KEYWORDS}

MTCMOS, sleep mode, leakage current, header switch, footer switch

\section{INTRODUCTION}

In the earlier days VLSI designers mainly concentrated on area, performance, speed, cost and reliability. But this performance improvement has lead to the increase in power dissipation. Reducing this power dissipation and achieving low power consumption has become a challenging task to the current day designers as cooling technology and packing are very expensive and also now a days because of the battery life time, the electronic circuit designers are worried about decreasing the total power consumption to increase the battery life time[11], especially for portable embedded systems and decrease the battery's size which is reflected on the portability of the devices. Power is very much concerned due to the remarkable growth and success of fields

Natarajan Meghanathan et al. (Eds) : ACITY, VLSI, AIAA, CNDC - 2016

pp. 41-51, 2016. ( ) CS \& IT-CSCP 2016

DOI : $10.5121 / \mathrm{csit} .2016 .60905$ 
like personal computing devices and wireless communication system which demand high speed computation and complex functionality with low power consumption .As the technology continue to scale down a significant portion of the total power consumption in high performance digital circuits is due to leakage current because of reduced threshold voltage[1]. MOSFETs are fabricated with high overall doping concentration, lowered source/drain junction depths, halo doping, high mobility channel materials, etc. Furthermore the reduction of the gate oxide thickness causes drastic increase in the gate tunnelling leakage current due to carriers tunnelling through the gate oxide, which is strong exponential function of the voltage magnitude across the gate oxide [2],[7]to minimize the leakage current. Here our main aim is to decrease the leakage current using MTCMOS technique.

\section{CMOS}

Complementary metal-oxide semiconductor is the most leading semiconductor technology used in the transistors that are manufactured into most of today's computer microchips. CMOS logic is well known for its extremely low static power dissipation and high noise immunity. CMOS is sometimes referred to as complementary-symmetry metal oxide semiconductor. Complementarysymmetry refers that a typical CMOS design style uses complementary and symmetrical pairs of p-type and n-type metal oxide field effect transistors for logic functions [8]. In CMOS technology, both kinds of transistors are used in a complementary way to form a current gate that forms an effective means of electrical control.

In this, all the PMOS devices will be together called as pull-up network and substrates are connected to the VDD, all the NMOS devices will be together called as pull-down network and its substrates are connected to the VSS. The output is taken at the centre as a function of inputs.

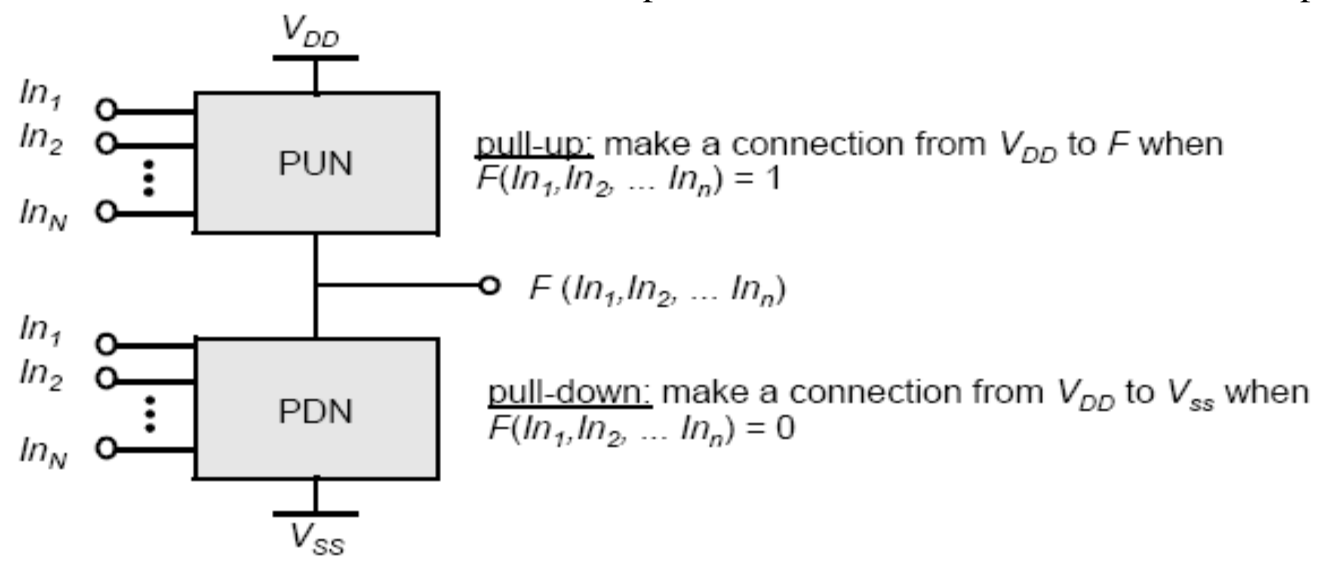

Figure 1. CMOS basic structure

\section{MTCMOS}

Multi-threshold CMOS is a power reduction technique, widely used in today's industry to lower the gate leakage current. The multi threshold CMOS technology has two main parts. First, "Active" and "sleep" operational modes [13] are associated with MTCMOS technology, for efficient power management. Second, two different threshold voltages are used for $\mathrm{N}$ channel and $\mathrm{P}$ channel MOSFET in a single chip [9]. The low threshold voltage transistor is able to switch 
faster but has a high leakage current when turned off compared to the high threshold voltage transistor which is slower to switch but has a low leakage current when off. In this we use low threshold transistor for logic and to separate it from power /ground with high threshold transistors and also the circuit is operated at high performances because of low threshold voltage transistor.

When a logic circuit is active, the sleep signals are de-asserted which turn on high threshold voltage transistors and create virtual VDD and GND around the logic. In inactive mode the sleep signals are asserted which separate the logic from the power/ground, there by lowers the leakage current. The MTCMOS technique shows no impact over circuit parameters such as output impedance, gain, threshold voltage, fluctuations and frequency response [10].

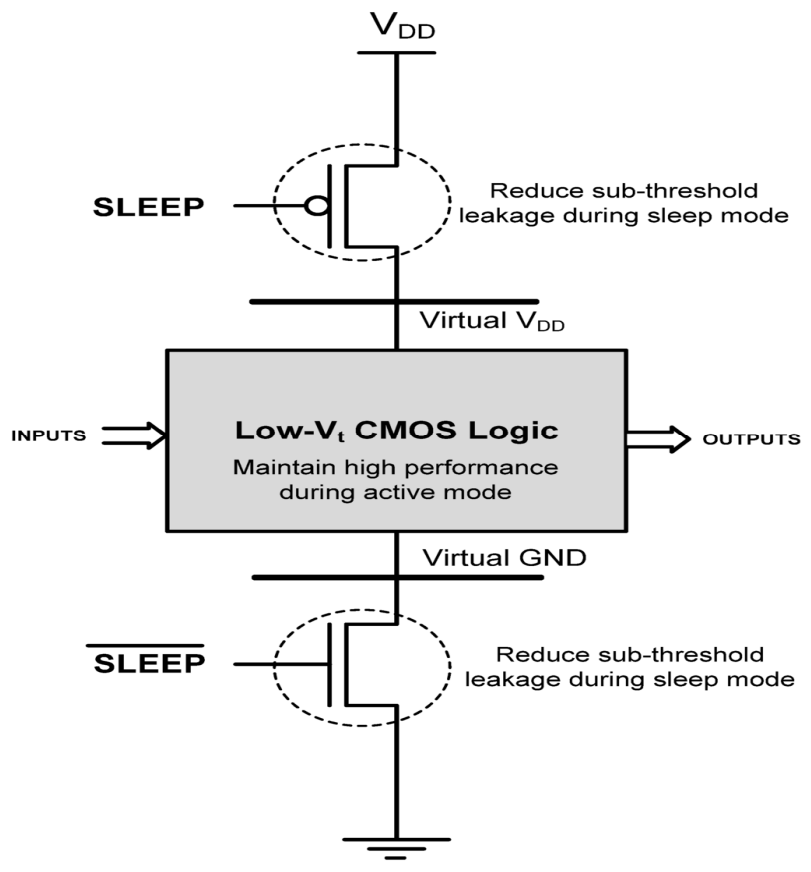

Figure 2. MTCMOS basic structure.

\section{POWER CONSUMPTION OF CMOS AND MTCMOS CiRCUITS}

The Complementary-Metal Oxide Semiconductor technology is well-known for its low power consumption. CMOS gates in older technologies were very efficient. In newer technologies, it has been skyrocketed due to transistor scaling, chip transistor counts and clock frequencies.

The average power over the time interval is

$$
\mathrm{P}_{\mathrm{avg}}=\mathrm{E} / \mathrm{T}=(1 / \mathrm{T}) \int_{0}^{\mathrm{T}} \mathrm{i}_{\mathrm{DD}}(\mathrm{t}) \mathrm{V}_{\mathrm{DD}} \mathrm{dt}
$$

Where $\mathrm{i}_{\mathrm{DD}}(\mathrm{t})$ is supply current and $\mathrm{V}_{\mathrm{DD}}$ is the supply voltage.

MTCMOS is a power gating technique in which a power gating transistor will be placed between the logic transistors and either powered or grounded, thus creating a virtual supply and virtual 
ground, respectively. Power gating is a technique used to reduce power consumption by shutting off the current to blocks of the circuit that are not in use. Lowering the threshold voltage results in an exponential increase in sub- threshold current [6]. As the circuit spends more time in the ideal (stand-by) mode, so it is practical to reduce the leakage current to minimize the static power which represents the dominant part of the total power consumption. Multi-threshold CMOS (MTCMOS) technology is one of the most effective techniques to reduce the leakage current during the standby mode by using a low threshold voltage transistor in the critical paths of the circuit to improve the performance while the high threshold voltage one is in uncritical paths and is used as an isolation switch between the virtual supply lines (VDD, GND) and the real one [14] . The high threshold voltage transistor is used for power consumption in the shortest path [3],[4]. Both active mode and sleep mode are associated for efficient power management.

\section{IMPLEMENTATION OF 2-BIT SERIAL IN SERIAL OUT SHIFT REGISTER}

A Shift Register is a sequential logic circuit that is used to store a sequence of data and this data is shifted by one clock pulse for every clock period at its output. They are a group of flip-flops that are connected in chain so that the output of one flip-flop will be given as the input to another flip-flop connected to it. All flip-flops are driven by a common clock and also they are set and reset simultaneously. Here we are taking both input and output in a serial manner so we call it as serial in serial out (SISO) shift register.

\subsection{Conventional 2-Bit SISO Shift register using CMOS}

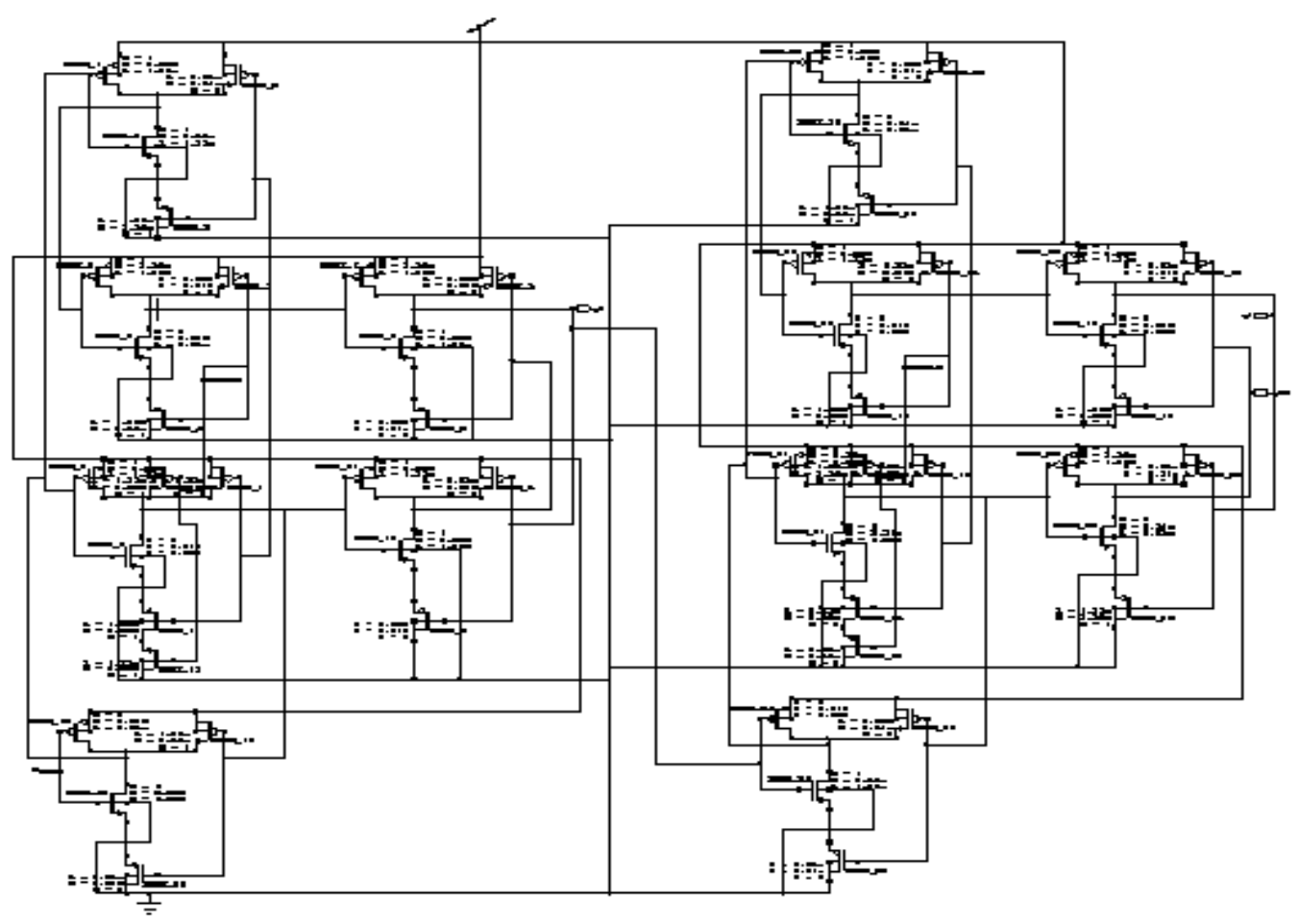

Figure 3. Schematic diagram of Conventional 2-Bit SISO Shift Register using CMOS 


\subsection{Simulation Result}

Taking $\mathrm{d}$ as the input, clock as the clock input,q1 as MSB and q0 as LSB we get the following output waveform for 2-Bit SISO Shift Register using normal CMOS technique.

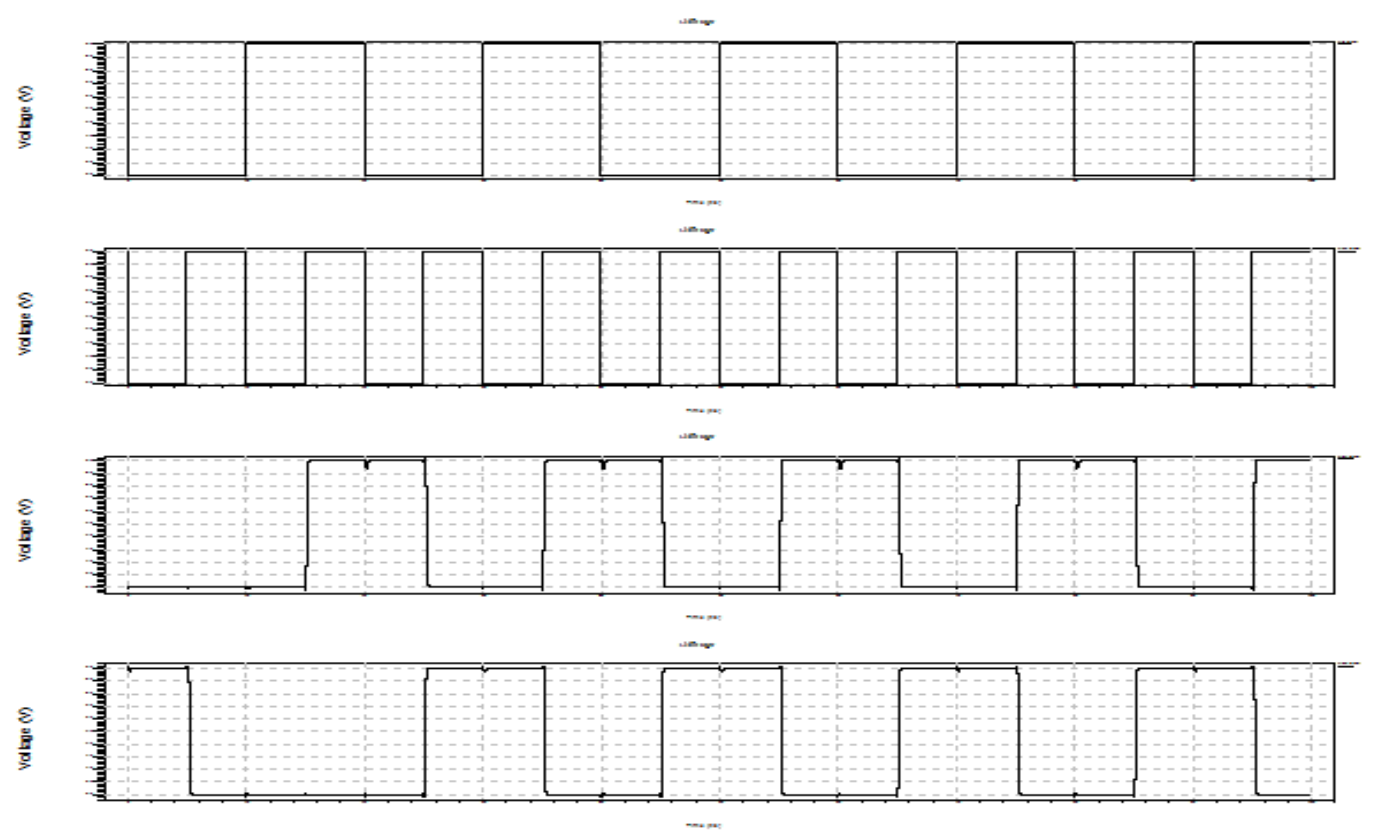

Figure 4. Output waveforms of conventional 2-Bit SISO Shift Register

\subsection{Proposed 2-Bit SISO Shift Register using MTCMOS technique}

To get the proposed design, we add a PMOS transistor that connects VDD and the circuit and forms a virtual power supply and a NMOS transistor that connects VSS and circuit and forms a virtual VSS. An inverter is designed, using it we give the sleep signal directly to PMOS and its inverted output to the NMOS.

The sleep transistor is controlled by a sleep signal that can be used to switch on and off the device. The PMOS sleep transistor can be called as "header switch" as it connects VDD supply to the circuit and the NMOS sleep transistor can be called as "footer switch" as it connects VSS supply to the circuit.

\subsection{Simulation Result}

Taking $\mathrm{d}$ as the input, clk as the clock input, $\mathrm{q} 1$ as the MSB and $\mathrm{q} 0$ as the LSB we get the following output waveform for 2-Bit SISO Shift Register using MTCMOS technique. 


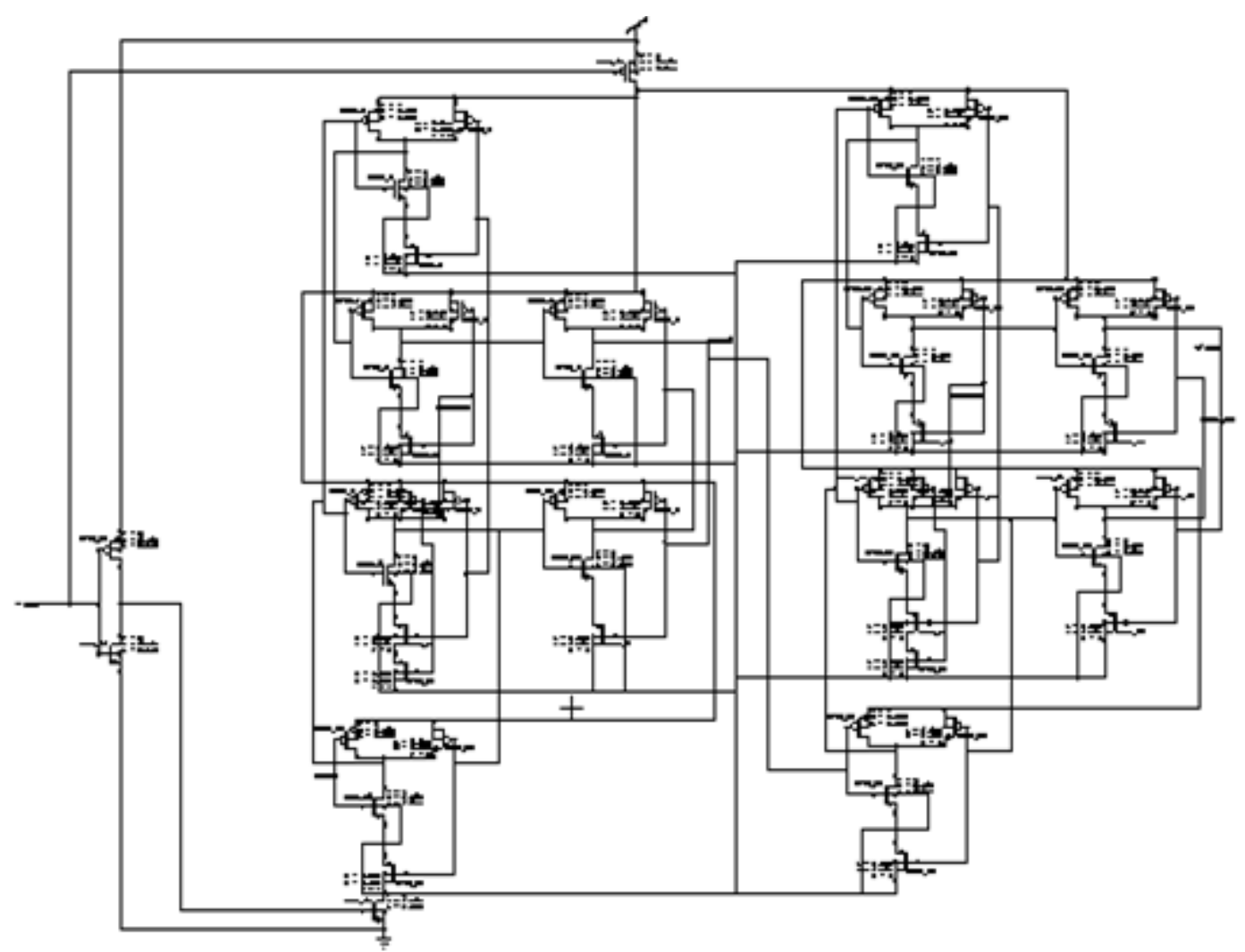

Figure 5. Schematic Diagram of Proposed 2-Bit SISO Shift Register using MTCMOS.

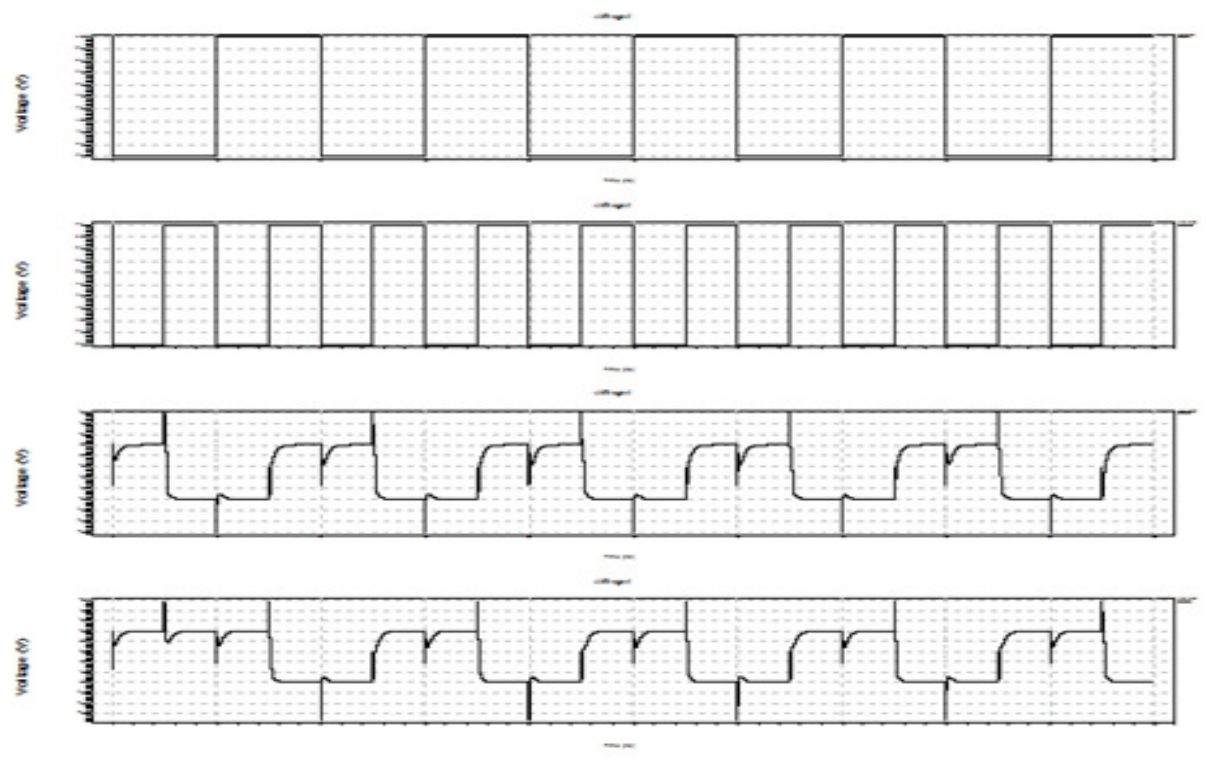

Figure 6. Output waveforms of proposed 2-Bit Shift Register.

\section{IMPLEMENTATION OF 2-BIT BINARY INCREMENTER}

The Binary Incrementer increases the value stored in the register by ' 1 '. For this implementation it simply adds ' 1 ' to the existing value stored in the register. For this implementation we need ' $n$ ' 
half adders to add ' $n$ ' number of bits. The storage capacity of the register is to be incremented. Here in the below example we are using two half adder to get 2-bit incrementer. The carry of the first half adder is used as input for the second half adder.

\subsection{Conventional 2-bit incrementer design using CMOS}

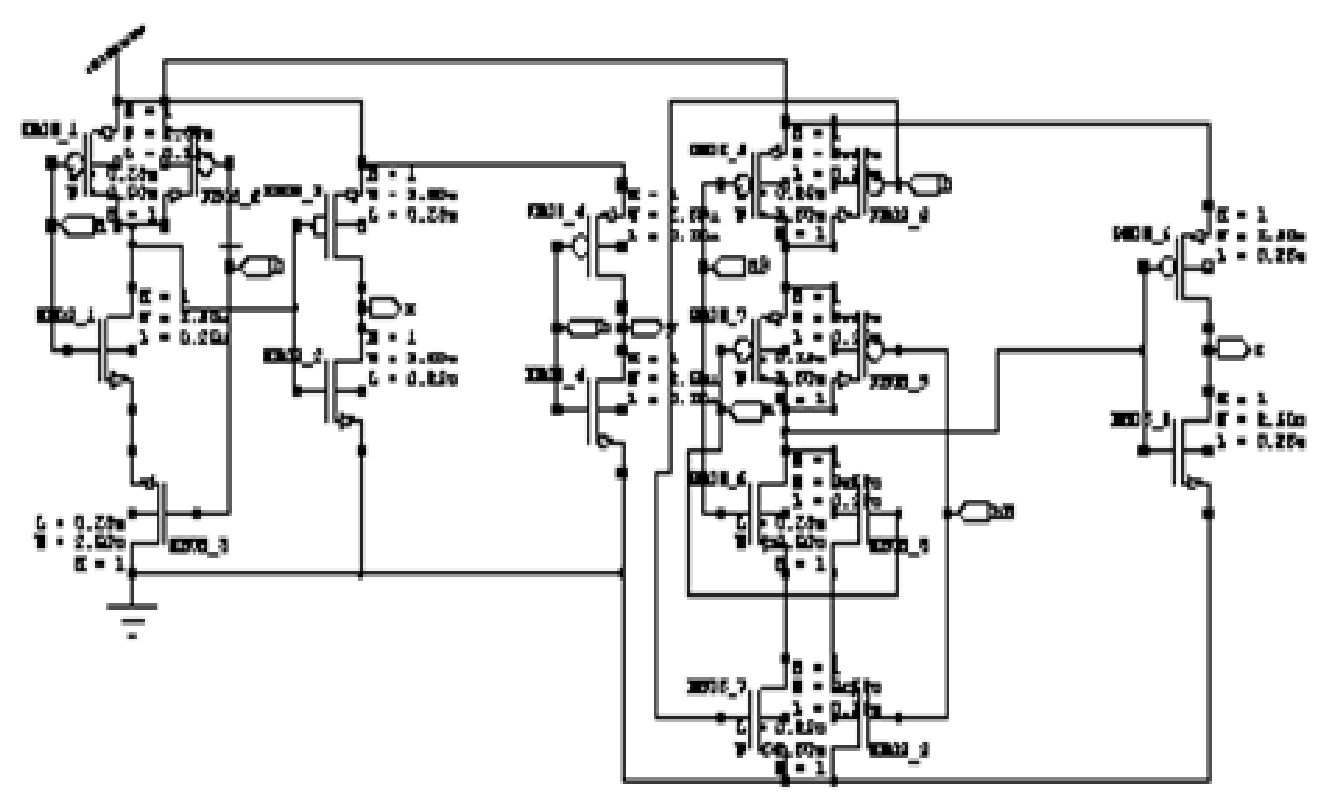

Figure 7. Schematic diagram of Conventional 2-bit binary incrementer using CMOS.

\subsection{Simulation Result}

Considering $\mathrm{a}, \mathrm{b}$ as the inputs and $\mathrm{x}, \mathrm{z}, \mathrm{y}$ as the outputs we get the following output waveforms for 2-bit binary incrementer using CMOS.

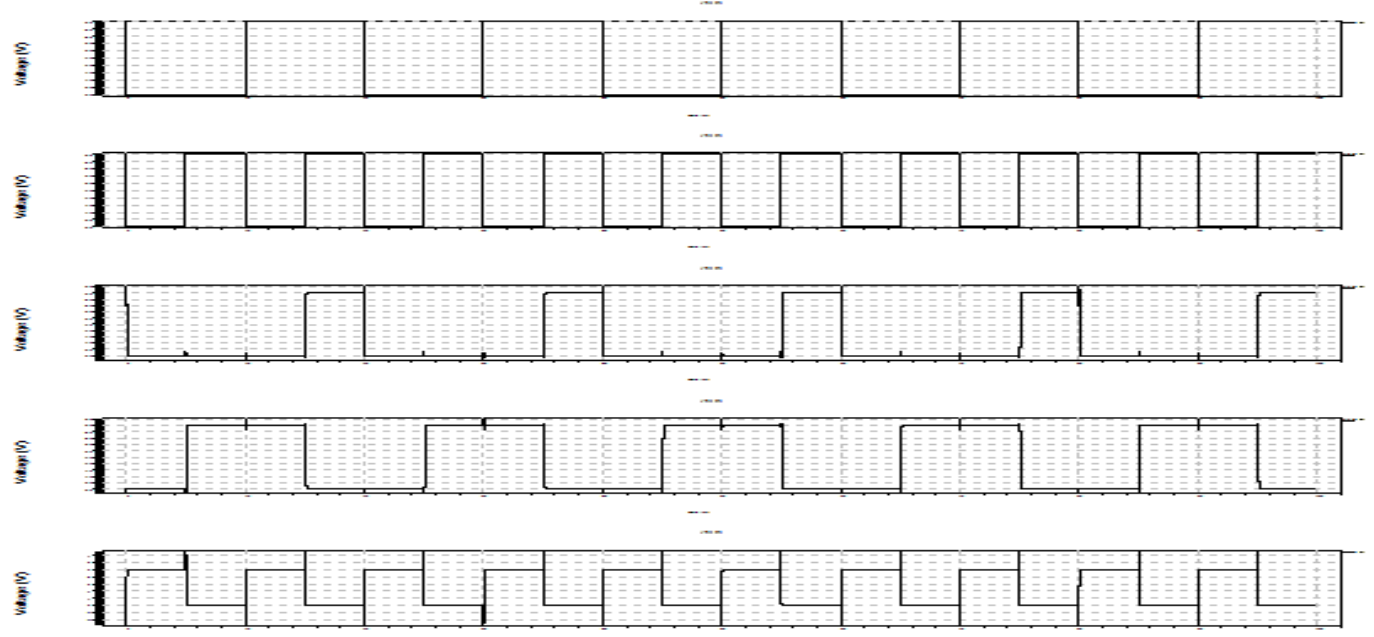

Figure 8. Output waveforms of conventional 2-bit binary incrementer using CMOS 


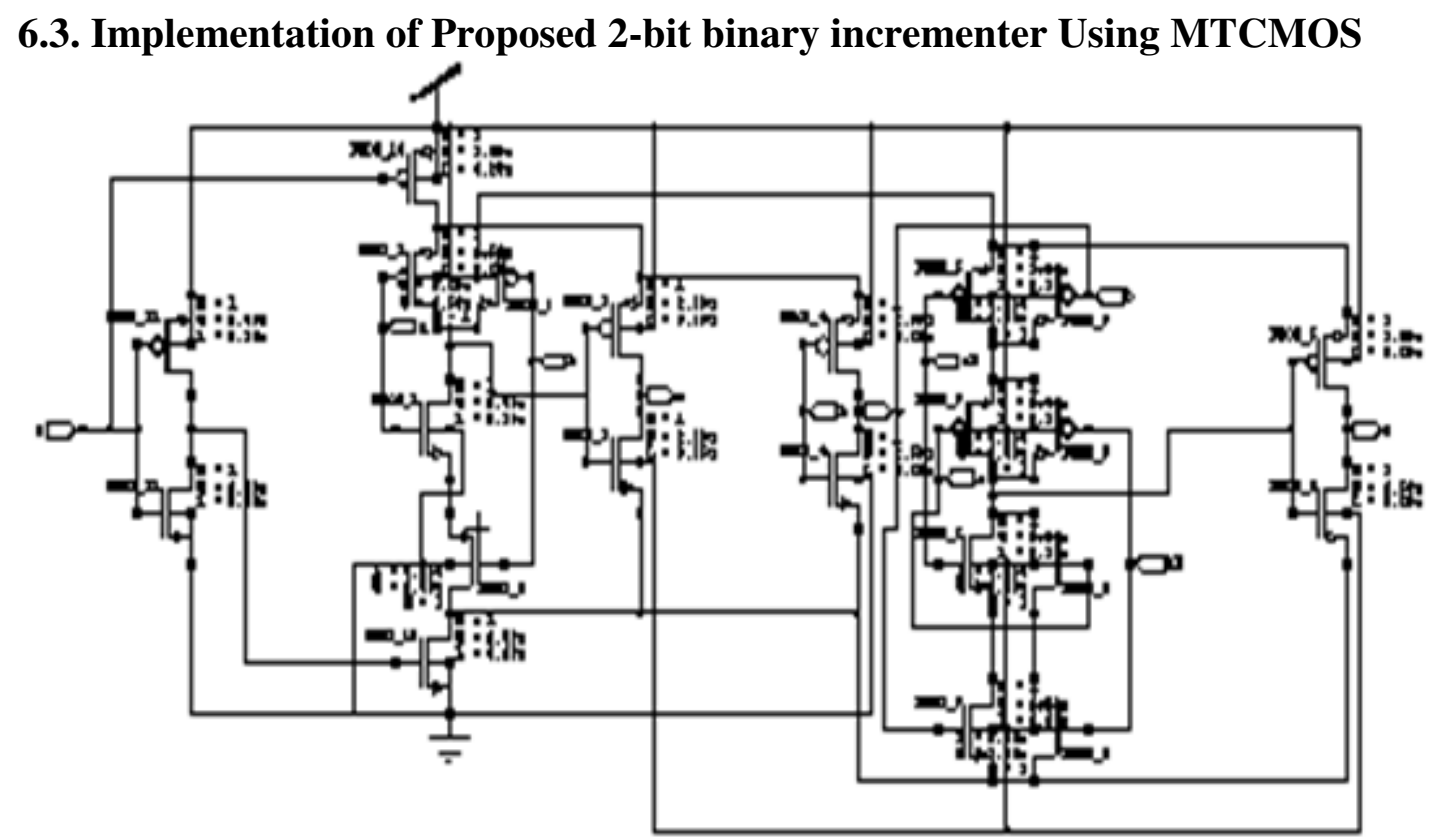

Figure 9. Schematic diagram of proposed 2-bit binary incrementer using MTCMOS

\subsection{Simulation Result}

Considering $\mathrm{a}, \mathrm{b}$ as the inputs $\mathrm{x}, \mathrm{z}, \mathrm{y}$ as the outputs we get the following output waveforms using MTCMOS technique.

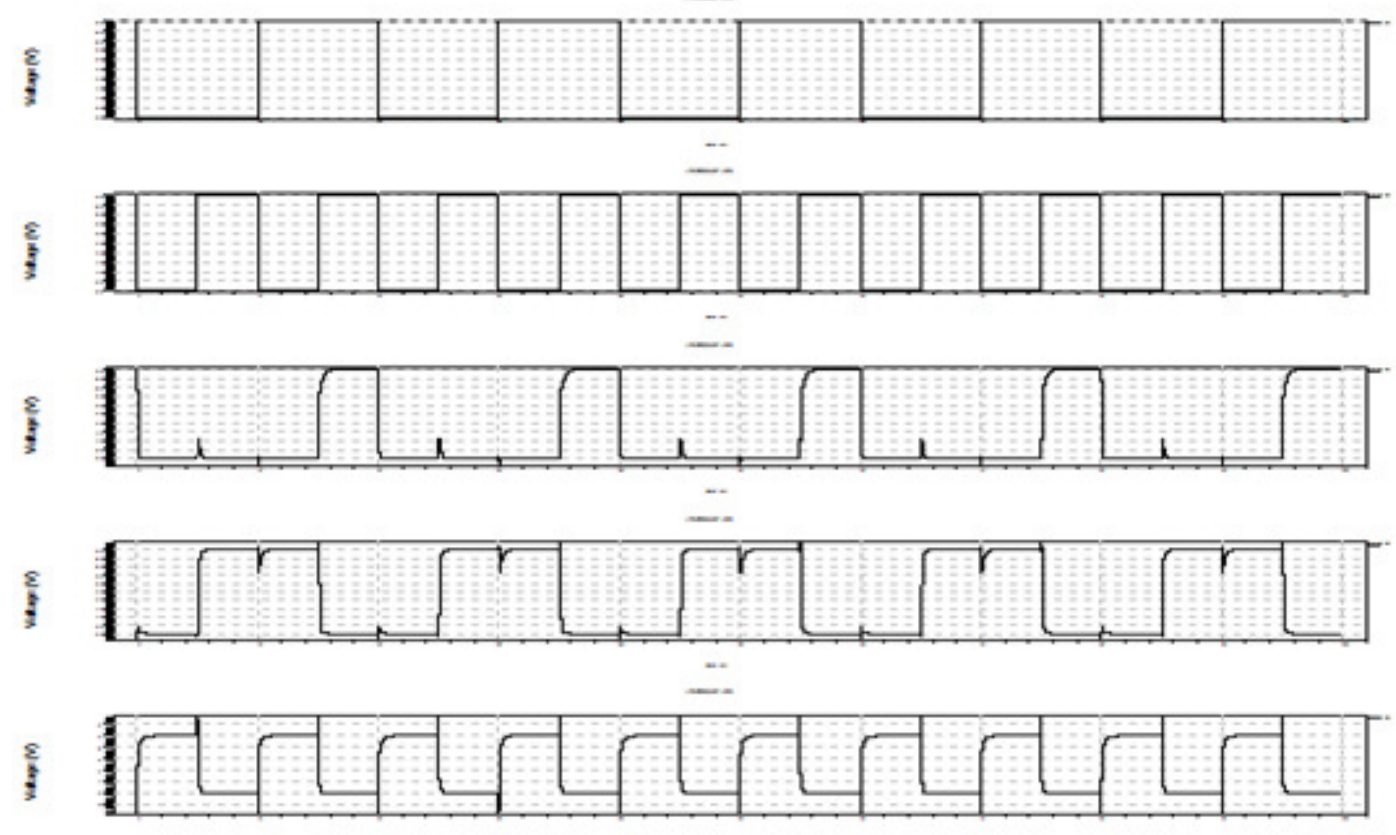

Figure 10.Output waveforms of proposed 2-bit binary incrementer 


\section{POWER CONSUMPTIONS OF 2-BIT INCREMENTER AND SHIFT REGISTER USING CMOS AND MTCMOS}

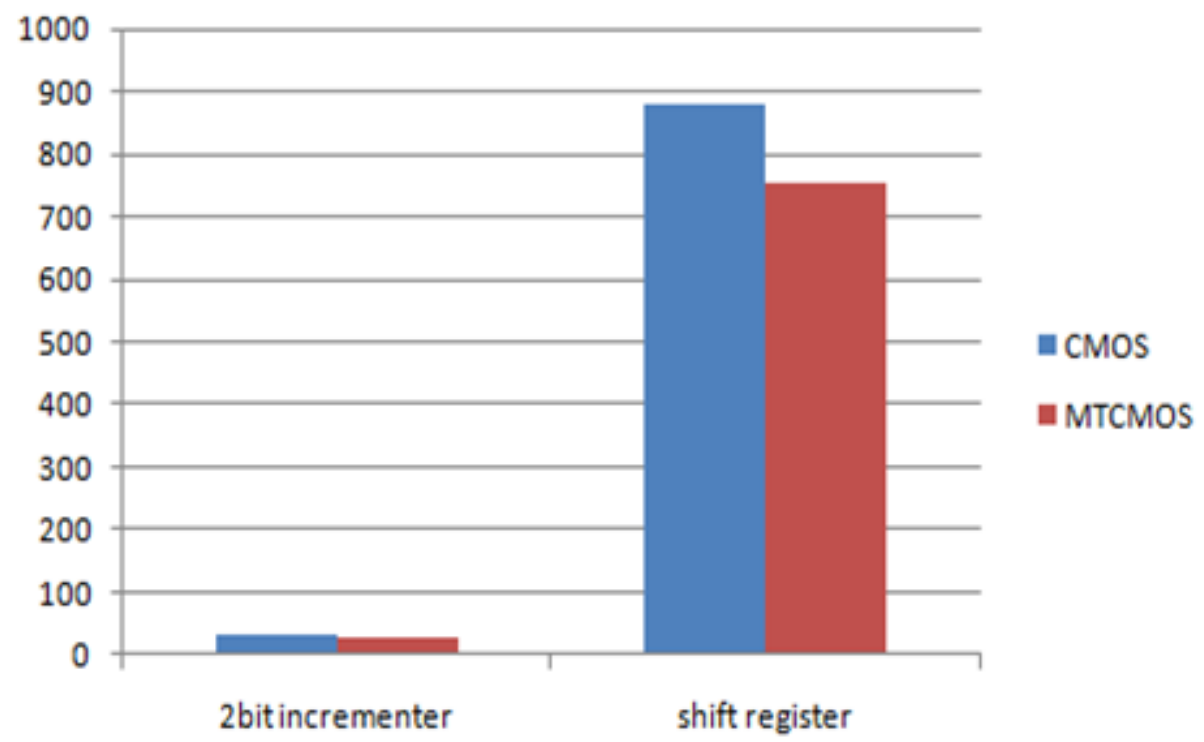

Figure 11. Graph of power consumptions of 2-bit incrementer and shift register

\subsection{Generalizing MTCMOS technique to different circuits}

Now apply the proposed MTCMOS technique to different kinds of gates and circuits and observe it's reduction in power consumption when compared to normal CMOS.

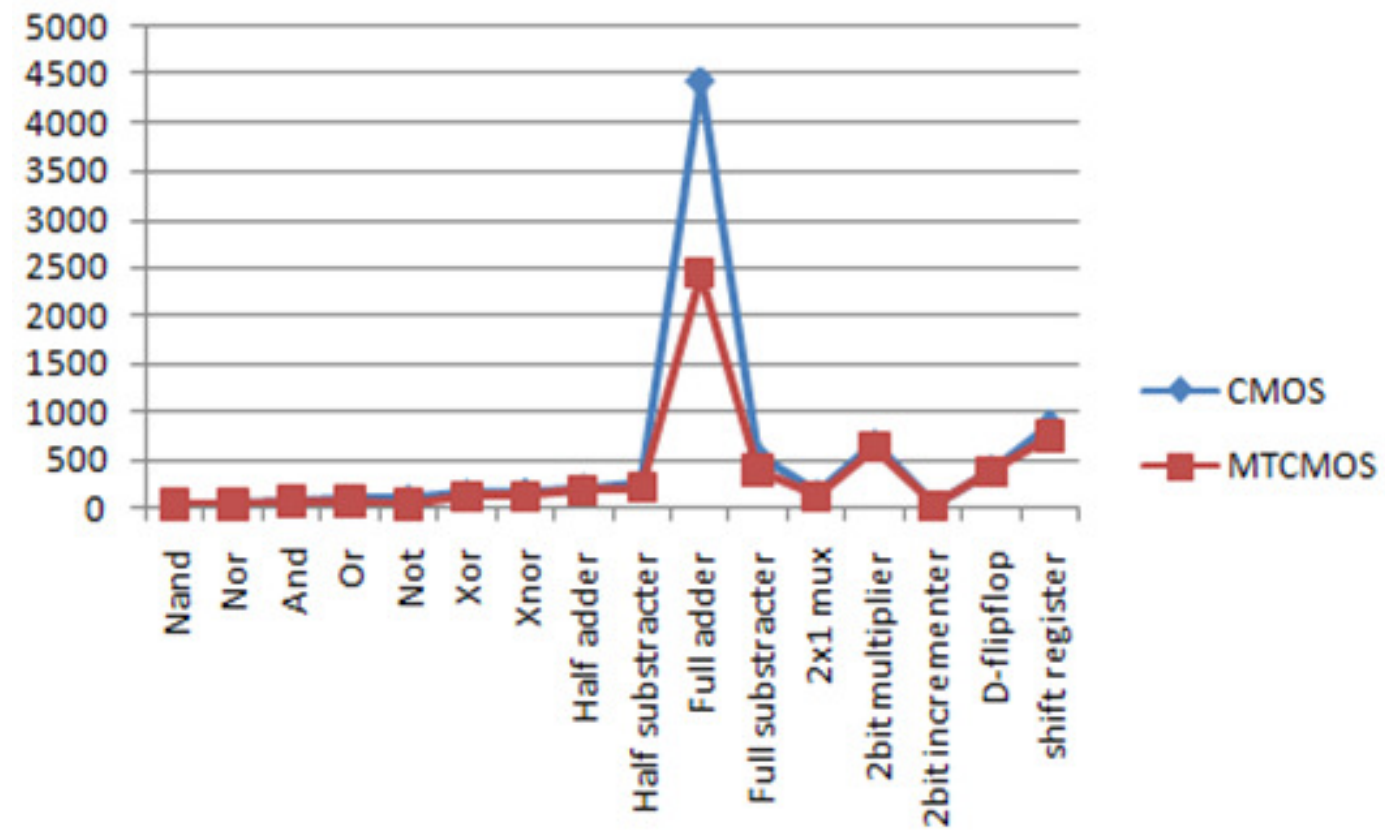

Figure 12. Difference in power consumptions between CMOS and MTCMOS Circuits 


\section{CONCLUSIONS}

In this paper we concentrated on the leakage current analysis and it can be reduced using the MTCMOS technique. The proposed technique is associated with different threshold transistors to build the CMOS circuits. All the circuits are designed using 250nm technology and operated with supply voltage. In this the total average power is decreased because of it's reduction in leakage currents using MTCMOS. As this technique deals with leakage current, we should always take care of the temperature.

\section{ACKNOWLEDGEMENTS}

The authors would like to thank Rajiv Gandhi University of Knowledge Technologies and National Institute of Science and Technology, Berhampur for providing their esteemed guidance and support to carry out their research work successfully.

\section{REFERENCES}

[1] Pawar Chander, Pokala Santhosh, Prasad Kurhe, "VLSI DESIGN OF FULL SUBTRACTOR USING MULTI-THRESHOLD CMOS TO REDUCE LEAKAGE CURRENT AND GROUND BOUNCE NOISE", ISSN, Volume-2, Issue-2, 2015.

[2] D. A. Antoniadis, I. Aberg, C. N. Chleirigh, O. M. Nayfeh, A. Khakifirooz and J. L.Hoyt, "Continuous MOSFET performance increase with device scaling: The role of strain and channel materialinnovations,” IBM. J. Res.Develop., vol. 50, no. 4, pp 363-376,Jul,2006.

[3] Dong Whee Kim, Jeong Beom Kee, "Low-Power Carry Look-Ahead Adder With Multi-Threshold Voltage CMOS Technology", in Proceeding of ICSICT International Conference on Solid-State and Integrated-Circuit Technolgy, pp.2160-2163,2008.

[4] H. Thapiliyal and N. Ranganathan, "ConservativeQCAGate (CQCA) for Designing Concurrently Testable Molecular QCA Circuits", Proc. Of the 22nd Intl. Conf.on VLSI Design, New Delhi, India, pp. 511-516, 2009.

[5] H. Thapiyal, M.B Srinivas and H.R.Arabnia, "Reversibile Logic Synthesis of Half, Full and ParallelSubtractors", Proc. Of the 2005 Intl. Conf. on Embedded Systems and Appilications, Las Vegas,pp. 165-181,2005.

[6] Hematha S , Dhawan A and Kar H , "Multithreshold CMOS Design for low power digital circuits" ,TENCON 2008-2008 IEEE Region 10 Conference,pp.1-5,2008.

[7] K. Roy, S. Mukhopadhyay , and H. Mahmoodi-Meimand, "Leakage current mechanisms and leakage reduction techniques in deepsubmicrometerCMOS circuits, "Proc. IEEE, vol.91, no. 2,pp. 305-327, Feb. 2003.

[8] “what is CMOS memory?” Wicked Sago. Retrieved 3 March. 2013.

[9] H.Thapliyal, M.B Srinivas, H.R Arabnia, "Reversible Logic Synthesis of Half, Full and ParallelSubtractors", proc. of the 2005 Intl. Conf. on Embedded Systems and Applications, Las Vegas, pp. 165-181. 
[10] Anis, M.H.; and Elmarsy, M.I. (2002). Power reduction via an MTCMOS implementation of MOS current mode logic. Proceedings of IEEE ASIC/SOC conference, 193-197.

[11] Itziar Marin, Eduardo Arceredillo, Jagoba Arias, Aitzol Zuloaga, Iker Losada, "low-power aware design: Topics on low battery consumption", proceedings of the 4th WSEAS Int. Conf. on Information Security, Communications and computers , Tenerife, Spain, December 16-18,2005(pp4752).

[12] A. Chilambuchelvan,S. Saravanan, B. Chidhambarar Ajan, J.Raja Paul Perinbam, "Certain Investigations on Energy saving techniques using DVS for low power embedded system", Proceedings of the 6th WSEAS International Conference on Applied Informatics and communications, Elounda, Greece, August 18-20,2006(pp298-305) .

[13] Alice Wang, Benton H. Calhoun, Anantha P. Chandrakasan, "subthreshold Design for Ultra LowPower Systems", Springers US, 2006.

[14] J.T. Kao, A. P. Chandrakasan, "Dual-threshold voltage techniques for low-power digital circuits", IEEE Journal of Solid-State Circuits, Volume 35, ISSUE 7, July 2000,pp. 1009-1018.

[15] K. Roy, "Leakage power reduction in low-voltage CMOS designs", IEEE International Conference on Circuits and Systems, Volume 2, Sept.1998, pp.167-173.

[16] B.H. Calhoun, F. A. Honore, A. p. Chandrakasan, “A leakage reduction methodology for distributed MTCMOS”, IEEE Journal of Solid-State Circuits ISSUE 5, May 2004, pp.818-826.

[17] Jan M.Rabaey, Anantha Chandrakasan, Borivoje Nikolic "Digital integrated circuits", a design perspective, second edition, 2003.

[18] Electronic Publication: Digital Object Identifiers(DOIs): ArtiCle in a journal:

[19] j. Clerk Maxwell, A. Treatise on Electricity and Magnetism, 3rd ed., vol.2. oxford: clarendon, 1892,pp.68-73.

[20] M. Young The Technical writer's Handbook. Mill Valley, CA: University Science, 1989. 